UDC 533.9

PACS 42.62.-b, 52.27.Ng, 52.35.Mw

DOI: $10.22363 / 2658-4670-2021-29-2-105-113$

\title{
On the possibility of averaging the equations of an electron motion in the intense laser radiation
}

\author{
Vladimir P. Milant'ev \\ Peoples' Friendship University of Russia (RUDN University) \\ 6, Miklukho-Maklaya St., Moscow 117198, Russian Federation
}

(received: March 12, 2021; accepted: May 25, 2021)

The problem of averaging of the relativistic motion equations of electron in the intense laser radiation, caused by the decreasing of the rate of wave phase change due to the Doppler's effect, is considered. As a result the phase can go from the "fast" to "slow" variables of the motion, so averaging over the phase becomes impossible. An analysis is presented of the conditions which are necessary for averaging of the relativistic equations of motion over the "fast" phase of the intense laser radiation on the base of the general principles of the averaging method. Laser radiation is considered in the paraxial approximation, where the ratio of the laser beam waist to the Rayleigh length is accepted as a small parameter. It is supposed that the laser pulse duration is of the order if the laser beam waist. In this case first-order corrections to the vectors of the laser pulse field should be taken into account. The general criterion for the possibility of the averaging of the relativistic motion equations of electron in the intense laser radiation is obtained. It is shown that an averaged description of the relativistic motion of an electron is possible in the case of a fairly moderate (relativistic) intensity and relatively wide laser beams. The known in the literature analogical criterion has been obtained earlier on the base of the numerical results.

Key words and phrases: intense laser pulse, relativistic electron, equations of motion, averaging of equations, criterion for averaged description of motion

\section{Introduction}

The nature of the motion of electrons in the field of electromagnetic waves substantially depends on the wave intensity, which is characterized by the dimensionless parameter $g=e E / \omega m_{e} c$. Here $E$ is the electric field amplitude of the wave, $\omega$ is its angular frequency, $e$ and $m_{e}$ are the electron charge and mass, respectively, $c$ is the velocity of light in vacuum. The first papers [1], [2] were devoted to the nonrelativistic motion of an electron in a high-frequency electromagnetic field of low intensity (parameter $g \ll 1$ ). It was shown by averaging over fast field oscillations, and expansions in terms of the parameter $g$,

(C) Milant'ev V.P., 2021

This work is licensed under a Creative Commons Attribution 4.0 International License

http://creativecommons.org/licenses/by/4.0/ 
that the particle was subjected to the action of an averaged (ponderomotive) force. Later, the relativistic generalization of the ponderomotive force was considered under the condition that the parameter $g$ was small [3], [4]. It was also noted that relativistic effects lead to various features of the averaged force [4]. In the field of high-power laser radiation, the parameter $g$ is large $(g \geqslant 1)$. So expansions in terms of the parameter $g$ become impossible. In the case of electrons, the parameter $g=1$, when the electric field strength $E_{r}(\mathrm{~V} / \mathrm{cm})=m_{e} c \omega / e=3.21 \cdot 10^{10} / \lambda(\mu m)$, where $\lambda(\mu m)$ is the wavelength. Radiation with electric field strength $E \geqslant E_{r}$ is called relativistically strong [5]. The parameter $g$ is commonly represented in the form:

$$
g=0.855 \cdot 10^{-9} \lambda \sqrt{I},
$$

where $I=\left(c E^{2} / 8 \pi\right)\left[\mathrm{W} / \mathrm{cm}^{2}\right]$ is the intensity of the laser pulse. The parameter $g$ is small in the case of a relatively weak field, when $I \ll I_{r}$. Here $I_{r} \equiv$ $m_{e}^{2} c^{3} \omega^{2} / 8 \pi e^{2}$ is the relativistic intensity determined by the electric field strength $E_{r}$. Intensity of modern lasers can reach $I \geqslant 10^{18} \mathrm{~W} / \mathrm{cm}^{2}$ [6]-[8].

In the study of particle motion, an adequate description of the laser radiation field plays an important role. When describing laser radiation, the paraxial approximation and its modifications is often used [9]-[13] which are based on the expansion of field vectors in terms of a small parameter

$$
\mu=a / Z_{R} \equiv 2 / k a \ll 1 .
$$

Here $a$ is the size of the laser beam in focus (beam waist), $Z_{R}=k a^{2} / 2$ is the Rayleigh length, $k=2 \pi / \lambda=\omega / c$ is the wave number. We assume that the laser field propagates in the $z$-direction. From the Maxwell equations, one can find the expressions for the transverse components of the radiation field vectors $\mathbf{E}_{\perp m}^{0}, \mathbf{B}_{\perp m}^{0}$ of the zero approximation in the form of Gaussian beams of various modes $m$ [9]-[13]. Longitudinal components $E_{z m}^{1}, B_{z m}^{1}$ also arise, which are of the first-order quantities. The parameter (2) establishes the relation between the wavelength of radiation and the size of the focal spot. Powerful laser radiation also has a characteristic scale - the length (or duration $\Delta t$ ) of the pulse. In the case of extended pulses, the corrections to the transverse components of the radiation field are second-order quantities [9]. If the pulse length $c \Delta t$ and the size of the focal spot $a$ are of the same order $c \Delta t \sim a$, then the first-order corrections to the transverse components of the field vectors $\mathbf{E}_{\perp m}^{1}, \mathbf{B}_{\perp m}^{1}$ appear [10]-[13]. In this case, the pulsed character of the radiation is specified by a fairly smooth pulse function $f(\sigma)$, where the parameter $\sigma=(t-z / c) / \Delta t$.

In the case of tightly focused laser radiation with the intensity $I \geqslant$ $10^{22} \mathrm{~W} / \mathrm{cm}^{2}$, the size of the focal spot can be equal to or smaller than the wavelength. In this case, the parameter (2) is not small, so that the paraxial approximation is not applicable and an exact solution of the Maxwell equations is necessary [14].

The presence of a small parameter (2) in the equations of the electron motion allows us to use the perturbation theory and perform averaging over fast oscillations of radiation. When they derive the ponderomotive force of a laser pulse, it is usually assumed that the wave amplitude varies slowly 
with respect to the wave phase (for example [15], [16]). However, the specific conditions for the relative change of these parameters are not considered. Meanwhile, the absence of such an analysis can lead to the misuse of averaging of the equations of motion. The fact is that during relativistic motion, the frequency of the radiation that the particle "sees" decreases due to the Doppler shift: $\omega^{\prime}=\omega\left(1-v_{z} / c\right)$. Here $v_{z}$ is the component of the particle velocity in the direction of the laser pulse propagation. Doppler frequency shift slows down the rate of wave phase change. Therefore, at a sufficiently high longitudinal velocity of the particle, the rate of phase change may turn out to be comparable with the change in the wave amplitude. This problem was partially touched upon in the paper [4]. However, the conditions under which the averaging of the equations of motion is permissible were not discussed in detail. It was verified in the work [10] by numerical calculations that the domain of validity of the averaged description of the electron motion in the ultraintense laser pulse was given by the condition $1-v_{z} / c \gg \varepsilon$, where $\varepsilon \equiv \mu / 2$. However, the meaning of this condition and its validity was not discussed.

This paper is devoted to the detailed analysis of the conditions for averaging the relativistic equations of the electron motion in the field of high-power laser radiation with a sufficiently long pulse duration such that $\lambda \ll c \Delta t \sim a$. In this case, the existence of a small parameter (2) is assumed as in the work [10].

\section{Basic relations}

The motion of an electron is described by the following equations:

$$
\begin{gathered}
\frac{d p_{x}}{d t}=-\left(1-v_{z} / c\right) e E_{x m}-e B_{z m}^{1} p_{y} / m_{e} c \gamma \\
\frac{d p_{y}}{d t}=-\left(1-v_{z} / c\right) e E_{y m}+e B_{z m}^{1} p_{x} / m_{e} c \gamma \\
\frac{d p_{z}}{d t}=-e E_{z m}^{1}-e\left(p_{x} E_{x m}+p_{y} E_{y m}\right) / m_{e} c \gamma \\
\frac{d \mathbf{r}}{d t}=\frac{\mathbf{p}}{m_{e} \gamma} \\
\frac{d \gamma}{d t}=-\frac{e}{\left(m_{e} c\right)^{2} \gamma} \mathbf{p E} .
\end{gathered}
$$

Here $\mathbf{p}=\left(p_{x}, p_{y}, p_{z}\right)$ is the electron momentum vector, $\gamma$ is the relativistic factor (dimensionless energy). The vectors of the laser field $\mathbf{E}_{m}, \mathbf{B}_{m}$ of an arbitrary mode $m$ taking into account first-order terms are determined by the formulas [13] (or [10]). So $\mathbf{E}_{m}=\mathbf{E}_{m}^{0}+\mathbf{E}_{m}^{1}$ and $\mathbf{B}_{m}=\mathbf{B}_{m}^{0}+\mathbf{B}_{m}^{1}$. Along with the equations (3), it is necessary to use the equation for the wave phase $\theta$ :

$$
\frac{d \theta}{d t}=-\omega\left(1-v_{z} / c\right) \equiv-\omega G / \gamma
$$


Here

$$
G \equiv \gamma-p_{z} / m_{e} c
$$

Let's note that the phase that the particle "senses" in the laser field differs from the phase $\theta$ by additional small terms [9]-[13]. However, in the case under consideration, these terms are not significant. The equations of motion (3) in the field of high-power laser radiation are very complicated for an analytical solution. Therefore, numerical methods of solution are often used that allow one to study some features of an electron motion in a laser field [10], [17]-[19]. In this case, most often, laser radiation is specified in the form of a Gaussian beam of the fundamental mode, even at $g \gg 1$, which, in general, is incorrect due to the following reasons: Solution of the Maxwell equations in the form of Gaussian (or Hermite-Gaussian) laser beams is the result of expansion of the field strength over the parameter (1). In the case of the ultra-intense and ultra-short laser pulses the size of the focal spot can be comparable with the wavelength [14]. So, the relation (1) is violated and description of the laser radiation in the form of the Gaussian beams becomes invalid. In this case exact solution of the Maxwell equations should be found [14].

\section{Conditions for relativistic equations of motion averaging}

A simplified description of electron interaction with a laser is achieved by averaging the equations of motion over the wave phase. Various versions of the averaged equations of motion have been considered in many works [20]$[24]$.

To average equations (3) over the phase $\theta$, it must be a "rapidly" changing quantity [25]. It follows from the equation (6) that this depends on the difference $1-v_{z} / c \equiv \Delta$, where $0<\Delta \leqslant 1$ (if the particle moves in the direction of wave propagation). At $\Delta \sim 1$ the phase changes "quickly" and averaging over the phase is possible. The last is a general condition for averaging the equations of motion. In the ultrarelativistic limit $(\Delta \ll 1)$ the phase $\theta$ becomes a "slow" (or "semi-fast") variable as well as the wave amplitude. In this case, the electron motion changes significantly and becomes more complicated [10]. In the presence of a unique small parameter (2), it is quite natural to present the above general averaging criterion in the modified form [10]:

$$
\Delta \sim 1 \gg \mu
$$

It follows from (6), that the difference $\Delta=G / \gamma$, where the quantity $G$, according to equations (3), satisfies the equation:

$$
\frac{d G}{d t}=e\left(1-v_{z} / c\right) E_{x m}^{1} / m_{e} c+\ldots
$$

One can see that the quantity $G$ is an integral of motion only in the case of a plane electromagnetic wave in vacuum $\left(E_{z m}^{1}=0\right)$. In this case, the value $G$ is determined by the initial conditions: $G=\gamma(0)-p_{z}(0) / m_{e} c$. If one considers the particles at rest at the initial instant of time, then $G=1$. In the case of laser radiation, the longitudinal field $E_{z m}^{1}$ always exists and plays 
an essential role in the motion of electrons. So, in general, the quantity $G$ contains a slowly changing part as well as quickly oscillating corrections with small amplitudes. However, it is sometimes believed that $G=1$ also in the case of laser radiation [11].

Let us further consider the averaging condition (8). In this case, the value $G$ can be represented as the expansion in terms of the parameter $\mu$ :

$$
G=G_{0}+G_{1}+\ldots,
$$

where $G_{0}$ (the averaged value of the quantity $G$ ) does not depend on the wave phase $\theta$, while $G_{i}$ are periodic functions. According to equation (9), the quantity $G_{0}$ remains constant up to the first-order terms. It follows from the condition (8) that averaging of the equations of motion is possible if the following inequality is fulfilled:

$$
\gamma \mu \ll G_{0} \sim 1
$$

or

$$
\gamma \ll 1 / \mu=\pi a / \lambda
$$

This condition must be satisfied both during the injection of particles in the radiation field, and during their further movement. It follows from (11), (12) that an averaged description of motion is allowed when the energy of an accelerating particle is limited. Typically [10] the parameter $\mu<6.4 \cdot 10^{-2}$. So the energy of the particle is restricted by the condition $\gamma \ll 17$.

Let us consider the relativistic factor $\gamma=\sqrt{1+p^{2} /(m c)^{2}}$. With the definition (7), it is easy to obtain the following equation:

$$
\gamma=\left[1+G^{2}+p_{\perp}^{2} /\left(m_{e} c\right)^{2}\right] / 2 G .
$$

Here $p_{\perp}^{2}=p_{x}^{2}+p_{y}^{2}$. It follows from the system of equations (3) that $p_{\perp} \sim g m_{e} c$. Then from (13), we obtain the following estimate: $\gamma \sim 1+g^{2} / 2$. Given the inequality (12), we conclude that the averaging of electron motion equations in the field of relativistically intense laser radiation is possible if a rather stringent condition is satisfied:

$$
1+g^{2} / 2 \ll \pi a / \lambda
$$

Thus, the averaging of the equations of motion is possible in the case of fairly moderate intensity of laser radiation and a relatively wide laser beam $(a / \lambda \gg 1)$. In the case of ultra-intense radiation $(g \gg 1)$, as it was already noted, the wavelength of the laser beam may be comparable with its size in the focus. Then the parameter in (2) turns out to be large, and expansion in the terms of this parameter becomes impossible. Moreover, at $g \gg 1$ the difference $1-v_{z} / c \cong\left[1+p_{\perp}^{2} /\left(m_{e} c\right)^{2}\right] / 2 \gamma^{2} \sim g^{-2} \ll 1$, and motion of an electron becomes very complicated as it was noted in the paper [10]. That means that the concept of the relativistic ponderomotive force has rather restricted domain of validity. 


\section{Conclusion}

It is shown that the condition $1-v_{z} / c \gg \mu / 2$, obtained by computer calculations in the paper [10], really corresponds to the general criterion (8) for averaging of the classical relativistic equations of electron motion in the intense laser beam. It leads to the conclusion that averaged description of the relativistic electron motion is possible at limited electron energy and limited intensity of the laser radiation, as it is established by the inequalities (12), (14). So averaging the equations of an electron motion over the wave phase seems to be possible in the case of a fairly moderate intensity and a relatively wide laser beam. Therefore, in general, it is impossible to consider the problem of the ponderomotive acceleration of electrons at very high intensity of the laser radiation. It should be particularly emphasized that for the averaging procedure it is necessary to take into account not only intensity but also other characteristics of the laser pulse.

\section{Acknowledgement}

The reported study was funded by RFBR, project No. 18-29-21041.

\section{References}

[1] H. Boot and R.-S. Harvie, "Charged particles in a non-uniform radiofrequency field," Nature, vol. 180, p. 1187, 1957. DOI: 10.1038/1801187a0.

[2] A. Gaponov and M. Miller, "Use of moving high-frequency potential wells for the acceleration of charged particles," Soviet Physics JETP-USSR, vol. 7, pp. 515-516, 1958.

[3] T. Kibble, "Mutual refraction of electrons and photons," Physical Review, vol. 150, p. 1060, 1966. DOI: 10.1103/PhysRev.150.1060.

[4] D. R. Bituk and M. V. Fedorov, "Relativistic ponderomotive forces," JETP, vol. 89, pp. 640-646, 4 1999. DOI: 10.1134/1.559024.

[5] G. A. Mourou, T. Tajima, and S. V. Bulanov, "Optics in the relativistic regime," Reviews of Modern Physics, vol. 78, p. 309, 2006. DOI: 10.1103/ RevModPhys.78.309.

[6] N. M. Naumova, J. A. Nees, and G. A. Mourou, "Relativistic attosecond physics," Physics of Plasmas, vol. 12, p. 056 707, 2005. DOI: 10.1063/1. 1880032.

[7] A. V. Korzhimanov et al., "Horizons of petawatt laser technology," Physics-Uspekhi, vol. 54, p. 9, 2011. DOI: 10.3367/UFNe.0181.201101c . 0009.

[8] C. Danson, D. Hillier, N. Hopps, and D. Neely, "Petawatt class lasers worldwide," High Power Laser Science and Engineering, vol. 3, 2015. DOI: 10.1017/hpl.2019.36.

[9] L. W. Davis, "Theory of electromagnetic beams," Physical Review A, vol. 19, pp. 1177-1179, 3 1979. DOI: 10.1103/PhysRevA.19.1177. 
[10] B. Quesnel and P. Mora, "Theory and simulation of the interaction of ultraintense laser pulses with electrons in vacuum," Physical Review E, vol. 58, pp. 3719-3732, 3 1998. DOI: 10.1103/PhysRevE.58.3719.

[11] G. V. Stupakov and M. Zolotorev, "Ponderomotive laser acceleration and focusing in vacuum for generation of attosecond electron bunches," Physical Review Letters, vol. 86, p. 5274, 2001. DOI: 10.1103/PhysRevLett. 86.5274.

[12] W. Wang, J. Xia L.and Xiong, H. Fang Z.and An, Z. Xie, W. Pei, and $\mathrm{S}$. Fu, "Field shaping and electron acceleration by center-depressed laser beams," Physics of Plasmas, vol. 26, p. 093 109, 2019. DOI: 10.1063/1. 5099508.

[13] V. P. Milant'ev, S. P. Karnilovich, and Y. N. Shaar, "Description of high-power laser radiation in the paraxial approximation," Quantum Electronics, vol. 45, pp. 1063-1068, 11 2015. DOI: 10.1070 / QE2015V045N11ABEH015800.

[14] S. G. Bochkarev and V. Y. Bychenkov, "Acceleration of electrons by tightly focused femtosecond laser pulses," Quantum Electronics, vol. 37, pp. 273-284, 3 2007. DOI: 10.1070/QE2007v037n03ABEH013462.

[15] E. Startsev and C. McKinstrie, "Multiple scale derivation of the relativistic ponderomotive force," Physical Review E, vol. 55, p. 7527, 1997. DOI: 10.1103/PhysRevE.55.7527.

[16] P. Mora and J. T. M. Antonsen, "Kinetic modeling of intense, short laser pulses propagating in tenuous plasmas," Physics of Plasmas, vol. 4, pp. 217-229, 1997. DOI: 10.1063/1.872134.

[17] Y. I. Salamin, G. R. Mocken, and C. H. Keitel, "Electron scattering and acceleration by a tightly focused laser beam," Physical Review Special Topics-Accelerators and Beams, vol. 5, p. $101301,2002$. DOI: 10.1103/PhysRevSTAB.5.101301.

[18] P. Wang et al., "Characteristics of laser-driven electron acceleration in vacuum," Journal of Applied Physics, vol. 91, pp. 856-866, 2002. DOI: 10.1063/1.1423394.

[19] A. Galkin, V. Korobkin, M. Y. Romanovsky, and O. Shiryaev, "Dynamics of an electron driven by relativistically intense laser radiation," Physics of Plasmas, vol. 15, p. 023 104, 2008. DOI: 10.1063/1.2839349.

[20] D. Bauer, P. Mulser, and W. H. Steeb, "Relativistic ponderomotive force, uphill acceleration and transition to chaos," Physical Review Letters, vol. 75, pp. 4622-4625, 25 1995. DOI: 10.1103/PhysRevLett.75.4622.

[21] I. Y. Dodin, N. J. Fisch, and G. M. Fraiman, "Drift lagrangian for a relativistic particle in an intense laser field," Journal of Experimental and Theoretical Physics Letters, vol. 78, pp. 202-206, 2003. DOI: 10 . 1134/1.1622032.

[22] N. B. Narozhny and M. S. Fofanov, "Scattering of relativistic electrons by a focused laser pulse," JETP, vol. 90, pp. 753-768, 5 2000. DOI: 10.1134/1.559160. 
[23] A. J. Castillo and V. P. Milant'ev, "Relativistic ponderomotive forces in the field of intense laser radiation," Technical Physics, vol. 59, pp. 12611266, 9 2014. DOI: 10.1134/S1063784214090138.

[24] V. P. Milant'ev and A. J. Castillo, "On the theory of the relativistic motion of a charged particle in the field of intense electromagnetic radiation," JETP, vol. 116, pp. 558-566, 4 2013. DOI: 10.1134 / S1063776113040067.

[25] N. N. Bogoljubov and Y. A. Mitropolskij, Asymptotic methods in the theory of nonlinear oscillations. New York: CRC Press, 1961, vol. 10, p. 537. DOI: $10.1007 / \mathrm{BF} 01056172$.

\section{For citation:}

V.P. Milant'ev, On the possibility of averaging the equations of an electron motion in the intense laser radiation, Discrete and Continuous Models and Applied Computational Science 29 (2) (2021) 105-113. DOI: 10.22363/26584670-2021-29-2-105-113.

\section{Information about the authors:}

Vladimir P. Milant'ev - Doctor of Physical and Mathematical Sciences, Professor of Institute of Physical Research and Technology of Peoples' Friendship University of Russia (RUDN University) (e-mail: milantiev-vp@rudn.ru, phone: + 7(499) 2483057, ORCID: https://orcid.org/0000-0003-4686-4229, ResearcherID: B-9335-2016, Scopus Author ID: 6602468700) 
УДК 533.9

PACS 42.62.-b, 52.27.Ng, 52.35.Mw

DOI: $10.22363 / 2658-4670-2021-29-2-105-113$

\title{
О возможности усреднения релятивистских уравнений движения электрона в поле мощного лазерного излучения
}

\author{
В. П. Милантьев \\ Российский университет дружбы народов \\ ул. Миклухо-Маклая, д. 6, Москва, 117198, Россия
}

Рассмотрена проблема усреднения релятивистских уравнений движения электрона в поле мощного лазерного излучения, вызванная уменьшением скорости изменения фазы волны из-за эффекта Доплера. Вследствие этого фаза может перейти из числа «быстрых» в число «медленных» переменных движения, так что усреднение по фазе становится невозможным. На основе общих принципов метода усреднения проведён анализ условий, при которых допустимо усреднение уравнений движения по «быстрой» фазе излучения. Лазерное излучение рассматривается в параксиальном приближении, в котором малым параметром является отношение сужения лазерного пучка к рэлеевской длине. Предполагается, что протяжённость импульса сопоставима с порядком сужения лазерного пучка. В этом случае необходимо учитывать поправки первого порядка к векторам поля лазерного импульса. Получен общий критерий, определяющий возможность усреднения релятивистских уравнений движения частицы в поле мощного лазерного излучения. Показано, что усреднённое описание релятивистского движения электрона возможно в случае достаточно умеренной (релятивистской) интенсивности и относительно широких лазерных пучков. Известный в литературе аналогичный критерий был получен ранее на основе численных расчётов.

Ключевые слова: мощный лазерный импульс, релятивистский электрон, уравнения движения, усреднение уравнений, критерий для усреднённого описания движения 